\title{
Enfer medades raras: enfermedades crónicas que requieren un nuevo enfoque sociosanitario
} Rare Diseases: chronic diseases that need a new approach

\author{
A. Avellaneda ${ }^{1-2}$, M. Izquierdo ${ }^{1-3}$, J . Torrent-Farnell ${ }^{4}$, J .R. Ramón ${ }^{1}$
}

\section{RESUMEN}

El concepto de Enfermedades Raras es relativamente nuevo. Suponen aquellos procesos “... que pueden ser mortales o provocar un debilitamiento crónico del paciente y que, debido a su escasa prevalencia, requieren esfuerzos combinados para tratarlas. A título indicativo, se considera una prevalencia escasa cuando es inferior a 5 casos por 10.000 personas en la Comunidad". La existencia de estas enfermedades está vinculada estrechamente con los medicamentos huérfanos, incluyendo como tales a todo fármaco, prótesis, agente biológico o preparación dietética destinado al tratamiento de una Enfermedad Rara. A este espectro hay que añadir dos factores más: 1. Los médicos de Atención Primaria no se sienten muy motivados en su conocimiento y 2 . Necesitan una atención sociosanitaria compleja y habitualmente más onerosa que las enfermedades crónicas. Por todo lo expuesto las Enfermedades Raras se presentan como un universo que requiere un nuevo enfoque sociosanitario por parte de los sistemas de salud.

Palabras clave. Enfermedades raras. Medicamentos huérfanos. Enfermedades crónicas. Modelo sociosanitario. Calidad de vida.

\begin{abstract}
The concept of Rare Diseases is relatively new. They are those processes "... that can be mortal or to cause a chronic weakening of the patient and who, due to its little prevalence, require combined efforts to treat them. For indicative purposes, a little prevalence is considered when is lesser than 5 cases per 10,000 people in the Community". The existence of these diseases is closely tied with orphan drugs, meaning all drugs, prosthesis, biological agents or dietetic preparations destined to the treatment of a Rare Disease. Besides, it is necessary to add two factors more: 1. The Primary Attention physicians do not feel very motivated in their knowledge and 2 . These diseases need a complex sociosanitary attention, habitually more expensive than chronic diseases.
\end{abstract}

By all exposed the Rare Diseases appear like a universe that requires a new sociosanitary approach from the health system.

Key words. Rare diseases. Orphan Drugs. Chronic Diseases. Sociosanitary Model. Quality of life.

An. Sist. Sanit. Navar. 2007; 30 (2): 177-190.

1. Instituto de Investigación de Enfermedades Raras (Instituto de Salud Carlos III).

2. Centro de Salud los Cármenes (Comunidad de Madrid).

3. Servicio de Análisis e Intervención en Salud Pública. Dirección General de Salud Pública (Comunidad de Madrid).

4. Fundación Dr. Robert-Universitat Autònoma de Barcelona.

\section{Correspondencia:}

Dr. J. R. Ramón

Instituto de Investigación de Enfermedades Raras

Instituto de Salud Carlos III

$\mathrm{C} /$ Sinesio Delgado, 6

28029 Madrid

Tfno. 918222909

E-mail: jramon@isciii.es 


\section{INTRODUCCIÓN}

El interés del hombre por las enfermedades es tan antiguo como la Historia de la Humanidad, sin embargo por motivos socioeconómicos evidentes, el objetivo de las diferentes sociedades ha sido atender prioritariamente los problemas relacionados con las enfermedades más comunes o prevalentes y con aquellas que generan una mayor morbi-mortalidad. Antes de la transición epidemiológica este interés se centraba en solucionar los problemas derivados de las enfermedades infecciosas, pero actualmente en los países occidentales la atención se centra en solucionar los problemas que generan las enfermedades crónicas, que adquieren una mayor relevancia con la consolidación del Estado de Bienestar, el progresivo aumento de la esperanza de vida y el consiguiente envejecimiento de la población.

El interés por las Enfermedades Raras (ER) surge en los países desarrollados. Aunque estas patologías no son específicas de estos países, sin embargo es únicamente en ellos donde los enfermos encuentran amparo para sus demandas asistenciales y sociales generadas, porque habitualmente se trata de enfermedades crónicas y con frecuencia invalidantes o con una mortalidad precoz elevada. Los problemas que generan están relacionados con el elevado coste social y sanitario que conlleva la ordenación y el estudio de este conjunto de enfermedades. Ante este panorama y con el nuevo escenario que supone la Ley de Promoción de la Autonomía Personal y Atención a las Personas en Situación de Dependencia ${ }^{1}$ es evidente que el enfoque sociosanitario que requieren estas enfermedades deberá ser revisado para hacer frente de forma integral y coherente a las necesidades de este colectivo desfavorecido².

\section{ENFERMEDADES CRÓNICAS}

Son muchas las definiciones que se han dado de enfermedad crónica (EC), pero quizás una de las más acertadas por su sencillez es la que hace $\mathrm{Nobel}^{3}$ en 1991 definiéndolas como un trastorno orgánico 0 funcional que obliga a una modificación del modo de vida del paciente y que persiste durante largo tiempo. Se trata por tanto de un problema que abarca a todos los grupos de edad, si bien afecta predominantemente a los adultos. Producen una limitación importante en la calidad de vida y el estado funcional de las personas que las padecen. Las enfermedades crónicas constituyen un desafío importante que ha debido asumir la salud pública en el último medio siglo4; son causa importante del aumento del coste sanitario, estimándose que en los países occidentales son responsables del $75 \%$ del gasto sanitario y de más del $80 \%$ del gasto farmacéutico ${ }^{5}$.

La medicina y más modernamente las ciencias de la salud se han ido interesando cada vez más por la evaluación de los aspectos que aparecen como consecuencia de la repercusión que la propia enfermedad tiene sobre los diferentes ámbitos de la vida del enfermo y que no pueden contemplarse tomando en consideración exclusivamente los signos y los síntomas que afectan al individuo enfermo, por lo que hoy en día se debe entender a la persona enferma en un marco holístico.

El paciente tiene que aprender a vivir con su dolencia crónica, adaptándose a su enfermedad para lograr el máximo de autonomía 
posible en su nueva situación. Éste es el reto del siglo XXI para el paciente crónico que supone un cambio de actitud en él para lograr una vida normal. Por tanto más que de un enfermo, se trataría de una persona con una salud diferenciada y que tras incorporar algunos cambios en su vida cotidiana se distinguiría de los no enfermos en la observación de sus síntomas y en la realización de controles médicos periódicos 6 .

\section{ENFERMEDADES RARAS}

Según la Unión Europea (UE) se entiende por Enfermedades Raras todas aquellas enfermedades

“...que pueden ser mortales o provocar un debilitamiento crónico del paciente y que, debido a su escasa prevalencia, requieren esfuerzos combinados para tratarlas. A título indicativo, se considera una prevalencia escasa cuando es inferior a 5 casos por 10.000 personas en la Comunidad"7.

Las ER en su mayoría son enfermedades crónicas que producen una gran morbilidad y mortalidad prematura, además de un alto grado de discapacidad y dependencia y, por tanto, un deterioro significativo de la calidad de vida de los afectados.

El término "raras", que se acuñó en torno a la prevalencia, no refleja la verdadera gravedad en términos de morbi-mortalidad y discapacidad, que además se desconoce en la mayoría de los casos. El criterio operativo de prevalencia, determinado por consenso, ni siquiera es homogéneo en todas las partes del mundo. Así, en Europa la cifra es de 5 por cada 10.000 habitantes, en Japón es de 4 por cada 10.000 habitantes, en EEUU la cifra es menos de 200.000 personas afectadas en el país (menor de 7,5 por cada 10.000 habitantes) ${ }^{8}$.

Esta definición no implica que el concepto sea meramente estadístico. Además últimamente se considera que la clasificación según la prevalencia no es un criterio muy adecuado, ya que para la mayoría de las $E R$, se desconoce el impacto y la carga de enfermedad que suponen éstas, y por otra parte tampoco se tiene conocimiento del tipo de discapacidades que conllevan. No se debe olvidar que el concepto de ER se ha acuñado como un término "paraguas", para facilitar la actuación en un grupo muy heterogéneo de enfermedades, en el cual se incluyen enfermedades cuya prevalencia es siempre baja pero en un espectro que abarca prevalencias muy dispares, de diversa etiología y difíciles de clasificar. Aparecen en grupos etarios muy diferentes y con frecuencia sin opciones terapéuticas curativas.

Si se considerara para su clasificación definitiva el aspecto meramente numérico, se ignoraría el aspecto nosológico o fisiopatológico, siendo por tanto un concepto relativo: así el síndrome metabólico sería una ER entre los aborígenes polinesios o africanos y no lo sería obviamente en la sociedad occidental, lo contrario sucedería con la malaria, la enfermedad más frecuente en el mundo y que genera mayor mortalidad. Se llegaría así a la difícilmente explicable situación de que en el mundo de la globalización existirían clasificaciones de enfermedades, como raras, diferentes en función de los continentes. Existen otros aspectos de la definición que deben subrayarse, en ese sentido y en un intento por caracterizar la problemática común a todas Ias ER, la UE incorpora además en su definición aspectos que 
se relacionan con la calidad de vida, la discapacidad y la carga de enfermedad ${ }^{7}$.

\section{MEDICAMENTOS HUÉRFANOS}

Uno de los problemas que plantean las ER es que debido a su extremadamente baja incidencia, la Industria Farmacéutica y los investigadores no han tenido estímulos en la búsqueda de fármacos para su tratamiento, utilizándose vicariamente medicamentos que alivien los síntomas.

En 1980 Althuis utilizó por primera vez el término de medicamento huérfano $(\mathrm{MH})^{9}$, para referirse a agentes con potencial terapéutico pero sin patrocinador para su comercialización. En 1983 se aprobó en Estados Unidos el "Orphan Drug Act"10, para promover la investigación y el desarrollo de este tipo de fármacos. Actualmente se define como $\mathrm{MH}$ todo fármaco, prótesis, agente biológico o preparación dietética destinado al tratamiento de una ER ${ }^{11}$.

En los últimos años se han hecho esfuerzos para promover el diseño y estudio de MH en USA (1983), J apón (1985), Australia (1997) y la UE (2000). Para promocionar su producción se ha estimulado: 1. la promoción de créditos tributarios y ayudas a la investigación, 2. simplificación de los procesos de autorización y 3. exclusividad en el mercado, aunque en la UE sólo se ha aplicado el último punto ${ }^{12}$. Con la aplicación de estos incentivos se ha conseguido un cierto avance en la investigación y desarrollo de nuevos $\mathrm{MH}$ aunque en el caso de la UE en menor proporción que en USA. Según Joppi y $\mathrm{col}^{13}$ el haber desarrollado sólo $18 \mathrm{MH}$ en 4 años de legislación puede ser debido a la escasez de los incentivos en la UE en comparación con otras legislaciones. En conclusión se necesitan más incentivos en Europa para desarrollar $\mathrm{MH}$ y desarrollarlos con coste adecuado ${ }^{12,13}$.

Gracias a la iniciativa del Comité de Medicamentos Huérfanos de la Agencia Europea del Medicamento, la característica de orfandad no debe ser entendida sólo en los fármacos de nuevo desarrollo, ya que define a los $\mathrm{MH}$ como aquellos que se utilizan en el tratamiento para una enfermedad rara ${ }^{12,14}$.

El desarrollo de la investigación farmacológica está haciendo posible descubrir nuevas indicaciones de utilidad para el tratamiento de ciertas ER de fármacos desarrollados y de utilidad demostrada para el tratamiento de enfermedades prevalentes, en ese caso no tendría sentido hablar de $\mathrm{MH}$, sino de indicación huérfana de un medicamento superándose así el concepto de uso compasivo, única forma de utilización hasta la fecha; así, el ibuprofeno, utilizado en el tratamiento del cierre del Ductus Arteriosus persistente, es una indicación huérfana, para el caso de este antiinflamatorio no esteroideo utilizado ampliamente en procesos inflamatorios, degenerativos, etc. ${ }^{12}$.

\section{MOVIMIENTO ASOCIATIVO}

Desde que el hombre primitivo sufrió por primera vez los sufrimientos de una enfermedad, recurrió a sus semejantes en busca de ayuda y éstos trataron de ofrecérsela según sus posibilidades. Con la evolución de la sociedad las llamadas redes de apoyo informal han ido diversificando su orientación desde la edad media en que tuvieron un carácter más benéfico religioso, pasando en la edad moderna 
por un movimiento obrero y reivindicativo de protección social del trabajador, hasta la sociedad actual en la que la mayor parte del tejido de estas redes se vertebra alrededor del movimiento asociativo ${ }^{15}$.

Con la llegada del Estado de Bienestar, a finales de los años ochenta en nuestro país, se produjo una importante movilización socio-política que supuso un gran empuje del movimiento ciudadano, destacando el protagonismo de la participación ciudadana como elemento de transformación social que presionó al Estado para que los cambios políticos se produjeran a mayor velocidad ${ }^{16,17}$. Se produce así el llamado "boom de la solidaridad"18. Apareció un interés por el voluntariado y el asociacionismo sin precedentes en la sociedad en general, que conjuntamente con el "boom de los medios de comunicación" hace posible que la sociedad civil comience a ser consciente de su importancia y aumente considerablemente la aparición del movimiento asociativo en los medios. Aparecen numerosos reportajes y artículos impensables hace sólo algunos años, que suponen un empuje al modelo asociativo, lo que facilita enfrentarse a los nuevos problemas, ofreciendo soluciones novedosas y creativas.

El movimiento asociativo actual se conforma como auténticos grupos de mentalización colectiva por lo que han logrado que determinados valores sociales, de difícil "venta" hace unos años, puedan ser asumidos como algo natural y normal por la población $n^{19,20}$.

En torno a la problemática compleja de las ER, en España, al igual que en el resto de países europeos, se ha desarrollado un amplio movimiento asociativo. Durante años las organizaciones o federaciones de pacientes con ER en todo el mundo han jugado un papel importante en el desarrollo de acciones orientadas a la mejora asistencial y de investigación en las ER. Se han esforzado en satisfacer las necesidades de información, centrando parte de su trabajo en la divulgación de la información existente, publicando páginas específicas en Internet o libros especialmente editados ${ }^{21,22}$. A través de esta meritoria tarea han informado básicamente a otros afectados de los problemas asistenciales y sociales que rodean a algunas de estas enfermedades o grupos de ER aproximándose a una descripción de las características principales de las mismas, así como de los recursos diagnósticos y terapéuticos fundamentales, aunque carezcan, en ocasiones, de una visión general del problema.

Es importante resaltar la existencia de las asociaciones de afectados, que forman una red que tiene entre otros objetivos la difusión de conocimientos de salud a la población, mejorando su entendimiento, ya sea mediante charlas, coloquios o con campañas publicitarias a nivel tanto local como general; para mejorar este aspecto podría ser interesante fomentar la creación de grupos de apoyo en las asociaciones de pacientes y reconocer su papel en la mejora del conocimiento y la difusión de las ER, lo que ayudaría a la integración familiar y social ${ }^{23}$.

No obstante, en España, el $88 \%$ del cuidado que precisa un enfermo con patología crónica lo prestan las redes sociales de apoyo, fundamentalmente la familia más cercana ${ }^{23-25}$. En estas redes se involucran un amplio espectro de personas, desde profesionales de la salud que ofertan cuidados intensivos en la asistencia de los pacientes hasta parientes o amigos que apoyan de una u otra forma el cuidado $0^{26,27}$. 
Al igual que en el resto de Europa, en España las asociaciones de pacientes han jugado un papel importante en la mejora asistencial y de investigación de las ER, proponiendo una estrategia común y el desarrollo de acciones orientadas a conseguir que se satisfagan sus necesidades.

Aunque en España el movimiento asociativo en las ER se inicia después que en otros países de nuestro entorno y con mayor "timidez", el aumento progresivo de las asociaciones y de los asociados, es indiscutible. El movimiento asociativo, está liderado, entre otras, por la Federación Española de Enfermedades Raras (FEDER), que desde su creación en el año 2000, está en rápido y continuo proceso de crecimiento y expansión: en Enero de 2006 estaba integrada por 104 asociaciones de afectados con un número aproximado de 15.000 personas asociadas, de las que según sus propias estimaciones al menos unos 9.000 eran enfermos. Existen actualmente más de 1.500 asociaciones de pacientes ${ }^{28}$; la base de datos del IIER (Instituto de Investigación en Enfermedades Raras) ${ }^{2,29}$ registra más de 600 asociaciones de ER en nuestro país, aunque muchas de ellas son pequeñas, centradas en enfermedades muy específicas y en muchos casos de carácter meramente local o regional. Intentan paliar las debilidades propias de su tamaño, escasez numérica de los afiliados, falta de recursos materiales y falta de recursos humanos adecuados, agrupándose en forma de alianzas o federaciones, aunque en ocasiones surjan conflictos de intereses y se encuentren con la competencia "desleal" de la federación a la que pertenecen, debidas en parte a la falta de "profesionalización" de las alianzas o federaciones ${ }^{30}$, a desconfianzas entre las asociaciones o a excesivos personalismos en los órganos directivos de unos y otros.

Por otra parte, con más frecuencia de lo deseable, las asociaciones comprueban que las alianzas o federaciones no cumplen las expectativas creadas, y que además éstas se ven favorecidas a la hora de recibir las subvenciones más cuantiosas, lo que hace que se mantengan nominalmente dentro de las federaciones 0 alianzas y sin embargo, adopten políticas en ocasiones contrarias a las actuaciones de las alianzas o federaciones a las que pertenecen.

El escenario actual, en el ámbito de las ER, nos brinda un movimiento asociativo muy atomizado y desunido, aunque aparentemente pueda parecer más o menos cohesionado en torno a diversas alianzas o federaciones.

La experiencia del movimiento asociativo internacional servirá sin duda de acicate para que las federaciones y organizaciones de pacientes en nuestro país consigan trabajar de manera más cohesionada y más eficiente con el objetivo de conseguir los objetivos y los retos que tienen planteados. En particular en la UE es de resaltar el papel de apoyo organizativo y estratégico que EURORDIS (European Organization for Rare Diseases) presta, de forma permanente, a las federaciones y alianzas nacionales de todos los países miembros.

\section{ENFOQUE SOCIOSANITARIO}

Afrontar las necesidades sociosanitarias es algo que se plantea cuando las sociedades alcanzan el Ilamado estado de bienestar. Éste contaba hasta ahora con tres pilares básicos: el Sistema de Pensiones, el Sistema Nacional de Salud y el Sistema Educativo; el Sistema 
Nacional de Dependencia, creado a partir de la entrada en vigor de la Ilamada La Ley de Promoción de la Autonomía Personal y Atención a las Personas en Situación de Dependencia, aspira a ser el 4ํㅜ pilar del Estado de Bienestar.

La Ley define la dependencia como:

"El estado de carácter permanente en que se encuentran las personas que, por razones ligadas a la falta o a la pérdida de autonomía física, intelectual o sensorial, precisan de la atención de otra u otras personas 0 ayudas importantes para realizar actividades básicas de la vida diaria."

El término dependencia lleva pues implícita la existencia de dos elementos: por un lado la persona dependiente y por otra la necesidad de atención a dicha persona. El fenómeno de la dependencia vinculado a la necesidad tanto individual como colectiva de hacer frente al menoscabo físico y psíquico de la persona, ha cobrado en los últimos años un auge especial adquiriendo la relevancia social que le corresponde.

Aunque las primeras referencias en la literatura a la dependencia son de finales de la década de los 60 del pasado siglo ${ }^{31,32}$, afrontarla no es una necesidad nueva, ya que las personas siempre han estado a riesgo de caer en una situación de dependencia; lo verdaderamente novedoso es la magnitud social que ha adquirido este problema. Hasta hace poco, el fenómeno de la dependencia se ha considerado implícitamente un problema privado que debía ser resuelto por las familias, responsables del "apoyo informal". Esta función ha recaído y recae, casi en exclusiva, en las mujeres de la familia y dentro de éstas, en las de mediana edad, entre 45 y 69 años por lo que tiene un indudable rol de género ${ }^{23,33}$.

La ley propone clasificar la dependencia en tres grados ${ }^{1}$ :

Grado I. Dependencia moderada: cuando la persona necesita ayuda para realizar varias actividades básicas de la vida diaria, al menos una vez al día.

Grado II. Dependencia severa: cuando la persona necesita ayuda para realizar varias actividades básicas de la vida diaria dos o tres veces al día, pero no requiere la presencia permanente de un cuidador.

Grado III. Gran dependencia: cuando la persona necesita ayuda para realizar varias actividades básicas de la vida diaria varias veces al día y, por su pérdida total de autonomía mental o física, necesita la presencia indispensable y continua de otra persona.

Además clasifica cada uno de los grados de dependencia en dos niveles, en función de la autonomía de las personas y de la intensidad del cuidado que requieran.

La dependencia puede clasificarse de distintas formas en función de su tipo:

Dependencia física: pérdida del control de sus funciones corporales y de su interacción con los elementos físicos del ambiente.

Dependencia social: asociada a la pérdida de personas y relaciones significativas para el individuo. 
Dependencia mental o cognitiva: cuando el individuo pierde su capacidad para resolver sus problemas y tomar decisiones propias.

Dependencia económica: tiene lugar cuando una persona pasa de ser un miembro "activo" económica y laboralmente, a formar parte de la población "inactiva", "pasiva" o "dependiente".

La dependencia es un fenómeno presente en todos los grupos de edad, si bien es cierto que se trata de un problema estrechamente vinculado al envejecimiento demográfico, por lo que se tiende a considerar que afecta sólo a los mayores, es decir existe una estrecha relación entre dependencia y edad. Las estimaciones para el año 2010, demuestran que el problema se agravará, incrementándose en unas 150.000 las personas dependientes mayores de 65 años, de las que un $50 \%$ serán mayores de 80 años $^{34.36}$ (Tabla 1).

Aunque en la actualidad el peso mayoritario de la dependencia corresponde a los mayores y dentro de ellos a los que superan los 80 años de edad (unas 440.000 personas en el año 2005), con frecuencia se "olvida" la dependencia vinculada a la discapacidad, baste citar como ejemplo la generada por las secuelas de accidentes laborales o de tráfico y la derivada de la presencia de enfermedad que no harán sino incrementar el problema, muy especialmente en el caso de las enfermedades crónicas.

La Encuesta sobre Discapacidades, Deficiencias y Estado de Salud (EDDS) realizada en 1999 y publicada por el Instituto Nacional de Estadística (INE) en el 2001, pone de manifiesto la existencia de una clara relación entre la salud, a nivel de las enfermedades crónicas y las situaciones de dependencia. En España la proporción de personas afectadas por enfermedades crónicas, por sexo y edad, presenta menor incidencia entre los menores de cinco años (10\%), mientras que en las edades más avanzadas alcanza a prácticamente el $85 \%$ de la población. Consideradas en su conjunto las enfermedades crónicas afectan al $44,2 \%$ de los hombres frente al $52,7 \%$ de las mujeres ${ }^{37}$ (Tabla 2 ).

Tabla 1. Estimación de la población dependiente $\geq 65$ años.

\begin{tabular}{ccccccc}
\hline \multirow{2}{*}{ AÑO } & \multicolumn{2}{c}{ Hombres } & \multicolumn{2}{c}{ Mujeres } & \multicolumn{2}{c}{ Total } \\
\cline { 2 - 7 } & $\geq 65$ años & $\geq 80$ años & $\geq 65$ años & $\geq 80$ años & $\geq 65$ años & $\geq 80$ años \\
\hline 1992 & 290.365 & 102.215 & 356.263 & 203.963 & 646.628 & 306.178 \\
2000 & 366.941 & 119.857 & 397.683 & 247.895 & 764.624 & 367.752 \\
2005 & 379.918 & 150.678 & 413.621 & 294.496 & 793.539 & 445.174 \\
2010 & 422.814 & 179.543 & 513.256 & 292.083 & 936.070 & 471.626 \\
\hline
\end{tabular}

Tabla 2. Prevalencia de padecer alguna enfermedad.

\begin{tabular}{lcc}
\hline & Hombres (\%) & Mujeres (\%) \\
\hline $0-19$ años & 22,9 & 21,2 \\
$20-44$ años & 35,1 & 42,8 \\
$45-64$ años & 60,6 & 71,2 \\
$>65$ años & 79,3 & 86,2 \\
Total & 44,2 & 52,7 \\
\hline
\end{tabular}


Según los datos de esta encuesta del total de la población que vive en hogares familiares, el $9 \%$ tienen algún tipo de discapacidad ${ }^{37}$; esta encuesta permite, en cierto modo, aproximarse al grado de dependencia de las personas adultas. Se podría estimar que entre los españoles mayores de 65 años: el 36,2\% presentaría una dependencia moderada; el 19,2\% presentaría una dependencia severa; el 17\% presentaría una gran dependencia.

Pero con los datos actuales no es posible obtener información sobre el grado de dependencia de los niños ni adolescentes, y esto es especialmente necesario en el caso de las ER que afectan mayoritariamente a la infancia.

Ante la nueva clasificación que propone la Ley de Dependencia serán necesarios nuevos enfoques en los estudios de investigación, ya que con los datos hasta ahora existentes únicamente se puede tener una visión cuantitativa de la frecuencia y distribución de la enfermedad crónica y de la discapacidad que ésta genera, pero no del grado de autonomía de las personas que las padecen. Evidentemente las ER deberán ser consideradas como un factor de peso, si se pretende enfocar la dependencia en su dimensión real, aunque en este caso se esté hablando mayoritariamente de personas jóvenes e incluso de niños, pero que deben convivir con diversas discapacidades de por vida.

Las intervenciones sociosanitarias ejercen un papel importante en el retraso en la aparición de la dependencia; intervenciones eficaces en edades tempranas de la vida pueden prevenir la aparición de la dependencia en edades más avanzadas. En el caso de las EC la mejora de los hábitos de vida, disminuyendo los hábitos que favorecen la dependencia: sedentarismo, sobrepeso, alcohol y tabaquismo, contribuyen significativamente a mejorar la esperanza de vida sin discapacidad ${ }^{34,35}$ y cuando la dependencia ya está presente, los cuidados sanitarios son esenciales tanto para la adecuada adaptación de la persona a esa situación, como para mejorar su calidad de vida. En el caso de las ER la intervención deberá dirigirse fundamentalmente a minimizar la dependencia a través de actuaciones integradas, vertebradas en torno al paciente y realizadas por equipos interdisciplinares, en el que han de participar obligatoriamente todos los niveles de atención, pero en los que el nivel primario de atención tanto sanitario como social deberán jugar un papel fundamental, ya que constituyen el entorno cercano al paciente.

\section{PAPEL DE LA ATENCIÓN PRIMARIA}

El papel del médico de atención primaria, no debe ser diferente al que tiene en las demás EC. Dado que en las ER se sabe que el diagnóstico precoz es vital para una evolución lo más confortable para el paciente ${ }^{38}$, el rol de la AP es fundamental a la hora de detectar signos precoces de alarma en los pacientes que sugieran este tipo de patologías, acelerando la visita a los especialistas adecuados para que el diagnóstico se realice con la máxima celeridad.

La relevancia de la función del médico de AP no se debe únicamente a este papel crucial en las primeras etapas de la enfermedad, aunque no concrete ni confirme el diagnóstico para lo que carece de medios técnicos, quedando en un segundo plano aparente ante la actuación de los especialistas, ya que la AP, desempeña o debe 
desempeñar un papel preponderante en el seguimiento, realizando una labor de "acompañamiento" del paciente, atento a sus necesidades y problemas asociados, valorando los signos de agravamiento del proceso y siendo un elemento clave en la coordinación de las actuaciones que se pongan en marcha para satisfacer la necesidades sociosanitarias de la persona ${ }^{39}$.

Un estudio cualitativo, recientemente llevado a cabo en $\mathrm{AP}^{40}$ no encontró diferencias entre los distintos profesionales (médicos, enfermeras y trabajadores sociales) en cuanto a su conocimiento de las ER o su interés hacia ellas, independientemente de que desarroIlaran su actividad en el medio rural o urbano. El citado estudio pone de manifiesto que el concepto de ER es poco conocido en AP. Dado que la mayoría de su actividad profesional se orienta a enfermedades prevalentes, les resulta sorprendente e incluso tienen dificultades para comprender que enfermedades de baja prevalencia puedan generar una problemática global de esta magnitud. Las ER despiertan escaso interés profesional, en contraste con el interés humano.

Una demanda frecuente por parte de los colectivos de pacientes e incluso de ciertos expertos en el ámbito de las ER es la mayor formación en las mismas de los médicos de AP. En contraste los profesionales entrevistados creen suficiente la formación que reciben en el pregrado y desestiman la formación de postgrado, por innecesaria y poco factible. Pero lo que reclaman de forma unánime es un acceso fácil y rápido a información contrastada sobre las mismas. Consideran la búsqueda de información activa, a través de Internet, como la mejor forma para obtener la información que optimice sus criterios de derivación y, en esa línea, el Sistema de Información de Enfermedades Raras en Español, SIERE (http://iier.isciii.es/er), es adecuado a sus demandas informativas.

\section{NUEVOS MODELOS DE ATENCIÓN}

La necesidad de plantearse un nuevo modelo surge porque el utilizado para la gestión en las enfermedades crónicas, no resulta útil en las ER; el modelo al uso no genera un verdadero dispositivo sociosanitario, salvo en casos excepcionales.

Se plantea pues la necesidad de un nuevo modelo de atención que articule un auténtico espacio sociosanitario que oriente y en lo posible mejore los diferentes problemas y consecuencias derivadas de estas enfermedades (discapacidad, dependencia, etc.). Por otra parte, el modelo debe tener en cuenta la pluralidad y heterogeneidad de las ER, además de la realidad administrativa de nuestro país, facilitando la gestión de las ER, reforzando los programas de coordinación y las políticas de equidad interterritorial. Debe entender y atender la complejidad de la situación de las personas con problemática sanitaria y social asociada situando en el centro a la persona, sus necesidades y el entorno social en el que vive.

El modelo de atención sociosanitaria requerido implica un cambio de cultura, ya que se orienta y vertebra, siguiendo las recomendaciones de la $\mathrm{OMS}^{41}$ (Tabla 3).

Un modelo que satisfaga las necesidades de los pacientes afectados por ER ha de integrar también como factores determinantes la atención especializada por la parte sanitaria, los llamados centros 
Tabla 3. Cambio de cultura en la articulación del modelo.

\begin{tabular}{|c|c|c|}
\hline Cultura & $\begin{array}{l}\text { Modelo sanitario } \\
\text { (enfoque actual) }\end{array}$ & $\begin{array}{l}\text { Modelo sociosanitario } \\
\text { (nuevo enfoque) }\end{array}$ \\
\hline Orientación & $\begin{array}{l}\text { Enfermedad } \\
\text { Diagnóstico } \\
\text { Tratamiento }\end{array}$ & $\begin{array}{c}\text { Cliente } \\
\text { Equipo interdisciplinar }\end{array}$ \\
\hline Cultura base & Hiperespecialización & $\begin{array}{c}\text { Valoración integral } \\
\text { Médica / funcional / social }\end{array}$ \\
\hline $\begin{array}{l}\text { Funcionalidad / } \\
\text { Discapacidad }\end{array}$ & No considerado & $\begin{array}{c}\text { Atención a discapacidad y } \\
\text { dependencia }\end{array}$ \\
\hline Organización & $\begin{array}{c}\text { Jerarquizado } \\
\text { Basado en diagnósticos } \\
\text { Estructural } \\
\text { Desintegrado }\end{array}$ & $\begin{array}{c}\text { Multidisciplinar } \\
\text { Basado en procesos } \\
\text { Funcional } \\
\text { Continuidad asistencial }\end{array}$ \\
\hline
\end{tabular}

base por la parte social y las asociaciones de pacientes por la parte comunitaria. Otros determinantes a incluir son: necesidad de información, autogestión de la propia enfermedad y atención a la dependencia.

Se ha constatado la necesidad de dar un paso más en el cambio de la mentalidad, ampliando el enfoque del curar por el de curar + cuidar, centrado en la valoración integral del usuario, donde la unidad de atención sea el usuario y su familia, que tenga en cuenta el control de la sintomatología, que considere los beneficios de la rehabilitación, que priorice la calidad de vida, que contemple la discapacidad y, potencie el trabajo en equipo para lograr un abordaje interdisciplinar.

\section{CONSIDERACIONES FINALES}

Las ER participan en gran manera de la evolución y de las necesidades de las enfermedades crónicas, aunque no todas Ias ER en la actualidad puedan considerarse crónicas (p. ej.: el labio leporino en general se trata al diagnosticarse, cosa que se hace al nacer o incluso previo al parto). Al igual que las $\mathrm{EC}$ necesitan atención médica y sociosanitaria específica, distinta de las enfermedades agudas, y más onerosa para el estado del bienestar; sin embargo, a diferencia de las enfermedades crónicas el médico de AP no está interesado en ellas, precisamente quizás por el hecho de su baja prevalencia ${ }^{40}$. Aspecto muy distinto es el del tratamiento: en las EC cada día la terapéutica es más sofisticada y elaborada como ocurre por ejemplo en la diabetes; por el contrario, hasta ahora en las ER el diseño de nuevas moléculas viene condicionado por la relación coste/beneficio, que a "priori" se supone que es baja. Una sociedad democrática e igualitaria, como la nuestra, no puede aceptar que les sean negados a ciertos ciudadanos los beneficios de los progresos médicos, por el hecho de que la enfermedad que sufren sólo afecte a un pequeño número de personas. Es competencia de la Administración proveer los incentivos y adaptar los procedimientos administrativos necesarios, para ofrecer a estos pacientes medicamentos con el mismo nivel de calidad, seguridad y eficacia de cualquier otro medicamento. Afortuna- 
damente las técnicas de genómica y el diagnóstico prenatal pueden Ilenar el vacío terapéutico a medio o largo plazo.

Es indudable que el componente operativo de la definición de ER ha resultado de un valor inestimable para hacer visibles estas enfermedades y sensibilizar tanto a la sociedad como a las autoridades sanitarias ante este problema relevante de salud pública y que resulta de innegable utilidad para resolver problemas en la investigación de las ER. Pero no es menos cierto que focalizar en este aspecto la gestión de estas enfermedades constituiría un error en el manejo global de las ER, cuyos problemas se deben intentar solucionar enmarcándolas en lo posible en las mismas clasificaciones y pautas de actuación que las restantes EC, pero reconociendo y actuando ante sus peculiaridades.

La participación del movimiento asociativo es sin duda un elemento clave en el enfoque sociosanitario que debe implantarse, ya que son los pacientes los verdaderos conocedores de la historia natural de su proceso y de la compleja problemática sociosanitaria que conlleva. El trabajo con las asociaciones debe garantizar la mutua colaboración en un marco de independencia; sin embargo la precariedad de la mayoría de estas asociaciones obliga a contribuir a su financiación por parte de la Administración, aunque el espectacular aumento de subvenciones de la Administración a las ONG ha incrementado su grado de dependencia al Estado, de tal forma que en ocasiones, se cuestiona el carácter no gubernamental ${ }^{42}$ de estas asociaciones.

Las ER plantean desafíos a la medicina actual muy distintos a los de hace sólo unos 30 años, que requieren para su solución abordajes novedosos y en los que desde la creación del IIER, el grupo de coordinación sociosanitaria está comprometido.

El cambio del modelo de atención a las EC priorizando un verdadero enfoque sociosanitario es una necesidad reconocida por todas las administraciones; pero como en toda situación de cambio, el desarrollo teórico ha ido muy por delante y la planificación teóricamente impecable, no se plasma en la realidad cotidiana. En la mayoría de las ocasiones, es en el núcleo familiar o en su entorno donde únicamente se materializa la coordinación sociosanitaria. En éste convergen los cuidados sociosanitarios al confluir las acciones de los profesionales de la salud, básicamente brindadas por la asistencia primaria, con la atención social que ofrecen las trabajadoras sociales, que desde hace unos años y de forma progresiva se integran en los Equipos de Atención Primaria.

Por esta razón nuestro grupo ha elaborado un nuevo modelo de atención sociosanitaria ${ }^{43}$ que intenta satisfacer las necesidades de atención de las enfermedades raras y que está resultando de utilidad. Aunque los primeros indicios parecen favorables, su verdadero nivel de coste-efectividad deberá ser testado a largo plazo.

\section{BIBLIOGRAFíA}

1. Ley 39/2006 de 14 de diciembre, de promoción de la autonomía personal y atención a las personas en situación de dependencia. BOE. 20056 Dec 14.

2. IzQuierdo M, Avellaneda A. Enfermedades Raras: Un enfoque práctico. 1a Edición. Madrid: ISCIII; 2004. 
3. Nobel G. Aspectos psicosociales del enfermo crónico. Enfermería psicosocial 1991; II: 239-241.

4. Serrano I. Cambios sociosanitarios del siglo XXI. La Educación para la Salud en el Siglo XXI. Comunicación y Salud. Madrid: Díaz de Santos; 2002.

5. García F, Sarría A. Revisión de intervenciones con nuevas tecnologías en el control de las enfermedades crónicas. Madrid: AETS-ISCIII; 2005.

6. PovedA R. Cuidados a pacientes crónicos. ANIORTE 2004. [consultado 2006]; URL disponible en:

http://perso.wanadoo.es/aniorte_nic/apunt_cuidad_cronic_1.htm

7. Decisión № 1295/1999/CE del Parlamento Europeo y del Consejo de 29 de abril de 1999. Diario Oficial de las Comunidades Europeas L 155/1. 1999.

8. Torrent-Farnell J, Morros R. The EU challenges on the designation of Orphan Medical Products. Pharmaceutical Policy and Law 2001; 3: 19-30.

9. Althuis TH. Orphan drugs-debunking a myth. N Engl J Med 1980; 303: 10041005.

10. The Orphan Drug Act. Public Law 1983; 4: 97-414.

11. Report of the National Commission on Orphan Diseses. Commission report Part I: Introduction. J Rare Diseases 1996; 2: 21-27.

12. Aronson JK. Rare diseases and orphan drugs. Br J Clin Pharmacol 2006; 61: 243-245.

13. J oppi R, Bertele V, GaratTinI S. Orphan drug development is progressing too slowly. Br J Clin Pharmacol 2006; 61: 355-360.

14. McCabe C, Tsuchiya A, Claxton K, Raftery J. Orphan drugs revisited. QJ M 2006; 99: 341-345.

15. IZQUIERDo M. Estrategias de Comunicación en Salud. Un ejemplo práctico: las Enfermedades Raras. Madrid: Tesis doctoral. Universidad Rey Juan Carlos. Madrid; 2005.

16. RodRíGUez G. La división social del bienestar: posibilidades y límites de la gestión mixta del Estado de Bienestar. Economía del sector no lucrativo Economistas. 1991; 51.

17. García X. El voluntariado en la sociedad del bienestar. Documentación Social 2001; 122: 15-39.

18. SALAMON L. The rise of the nonprofit sector. Foreign Affairs 1994; 74.

19. ALONSO LE. Nuevos movimientos sociales y asociacionismo. Institucionalización, estructura económica y desarrollo asociativo. Las entidades voluntarias en España. Ministerio de Asuntos Sociales. Madrid. 1996.

20. Zubero I. Movimientos sociales: mito, realidad, alternativa. 25 - 27 de noviembre. Madrid: Acta del V Congreso Estatal de Intervención Social; 1998.

21. Ayme S, Oziel D, Urbero B, Lecouturier E, Biscarat aC. Information sur les maladies rares: le projet Orphanet. Rev Med Interne 1998; 19 Suppl 3: 376377.

22. Hunting out rare diseases on the Web. Trends Endocrinol Metab 2000; 11: 251.

23. García-Calvente Mm, Mateo-Rodríguez I, Gutiérrez P. Cuidados y cuidadores en el sistema informal de salud. Granada: Escuela Andaluza de Salud Pública; 1999.

24. DuRÁn MA. El tiempo y la economía española. ICE 1991; 695: 9-48.

25. J ORDAN B. Value for caring. London: King Edward's Hospital Fund for London; 1990.

26. Molina J M, IÁÑEZ MA, láÑEZ B. El apoyo social como modulador de la carga del cuidador de enfermos de Alzheimer. Psicología y Salud 2005; 15: 33-43. 
27. SPITZE G, Logan JR, Robinson J. Family structures and change in living arrangements among elderly no married parents. J Gerontol 1992; 47: 289-296.

28. Fundación FARMAINDUSTRIA. EI paciente en España. Mapa nacional de asociaciones de pacientes. Fundación FARMAINDUSTRIA 2004. [consultado 2006]; URL disponible en:

http://www.farmaindustria.es/farmaweb/7pb43811prod.nsf/9EE803F8F46

29. SIERE (Sistema de Información de Enfermedades Raras en Español). IIERISCIII (MSC) 2006. URL disponible en: http://iier.isciii.es/er

30. J OHNSTON H, LARAÑA E, GUSFIELD J I Identidades, ideologías y vida cotidiana en los nuevos movimientos sociales. En: Laraña E, Gusfield J, editores. Los nuevos movimientos sociales, de la ideología a la identidad. Madrid: CIS; 1994.

31. USDHEW. Toward a social report. Washington DC: U S Department of Health, Education and Welfare (USDHEW); 1969.

32. OCDE. Liste des preocupations sociales communes à la plupart des pays de I'OCDE. Paris; 1973.

33. García-Calvente Mm, Mateo-Rodríguez I, Eguiguren AP. El sistema informal de cuidados en clave de desigualdad. Gac Sanit 2004; 18 (Supl.1):132-139.

34. Suzman RM, Manton KG, Willis DP. Introducing the oldest old. En: Suzman RM, Willis D P, Manton K G, editores. The Oldest old. New York: Oxford University Press; 1992.

35. Manton KG, Stallard E, Tolley HD. Limits to human life expectancy: evidence, prospect and implications. Popul Dev Rev 1991; 17: 603-637.

36. Rodríguez G. La protección social de la dependencia en España. Madrid: IMSERSO; 2004.

37. Instituto Nacional de Estadística (INE), Instituto de Migraciones y Servicios Sociales (IMSERSO), Fundación ONCE. Encuesta sobre Discapacidades, Deficiencias y Estado de Salud (EDDS). INE 2001. [consultado 2006]; URL disponible en:

http://www.ine.es/prodyser/pubweb/disc_inf05/discapa_inf.htm

38. García-Ribes M, Gaite L. El medico de familia ante las enfermedades raras: un nuevo desafío para la coordinación asistencial. Aten Primaria 2005; 36: 243.

39. Servicio de Pediatría Hospital Universitario "Marqués de Valdecilla", Universidad de Cantabria. Necesidades de los pacientes pediátricos con enfermedades raras y de sus familias en Cantabria. Documentos 69/2005. Real Patronato sobre Discapacidad. Ministerio de Trabajo y Asuntos Sociales. 2005.

40. Avellaneda A, Izquierdo M, Luengo S, Arenas J, Ramón JR. Necesidades de formación en enfermedades raras para atención primaria. Aten Primaria 2006; 38: 345-348.

41. OMS. Cuidados innovadores para Condiciones Crónicas. OMS. 2003.

42. Rivera J. Un análisis de los grupos de ayuda mutua y el movimiento asociativo en el ámbito de la salud: adicciones y enfermedades crónicas. R A E. 2005; 5 (Texto 13).

43. Avellaneda A. Atención sociosanitaria en las Enfermedades Raras: un modelo para las enfermedades crónicas discapacitantes. Madrid: Tesis doctoral. Universidad Rey Juan Carlos. Madrid; 2007. 